\title{
Application of WSN for Smart Power Metering to Avoid Cheating on Electric Power Consumption at Places with Shared Power Sources
}

\author{
Michal Hodoň \\ University of Žilina, Univerzitná 8215/1, \\ 01026 Žilina, Slovakia, Email: \\ michal.hodon@fri.uniza.sk
}

\begin{abstract}
Application scenarios of WSN implementations are really broad. Due to their typical characteristics, such advantageous power consumption, wide communication coverage and relatively broad sensing possibilities, many application fields can be covered. In this paper, the problematic of power metering is targeted. Places with shared power sources, where electricity usage is calculated equally according to the number of users, are often misused by single users for illegitimate power usage. Special WSN was developed and implemented in a scenario of multi-store parking garage to solve this problem.
\end{abstract}

\section{INTRODUCTION}

$\mathrm{W}$ ireless sensor networks (WSNs), as the front-end representative of Internet-of-Things framework, are still more and more implemented in real applications of our present life. When considering the typical WSNs implementation advantages, as their scalability, capability, integration simplicity or operability easiness, their application usage is really broad. The typical applications cover mainly the problematic of environment monitoring [1], [2], [3], e.g. with particular focus on the specific parameters of the nature [4], [5], [6], [7]. Other typical abundant application field is the area of Intelligent Transportation systems, where WSNs already got used to the climate, see [8], [9], [10], [11]. Secondary areas, with no such a high WSN implementation capabilities, are military [12] and eHealth [13], where some strict security/safety rules make the accommodation of central applications difficult. However, by implementation of specific approaches, as [14], [15], [16], [17] and [18], the application fields covered by WSNs could be wider, e.g. agriculture, industry, home automation... Especially the last two areas offer interesting opportunities of WSN indoor implementations, the application problematic on which this paper is geared to.

Real applications of WSNs indoors are the challenging tasks, which due to the certain specific conditions - stronger multipath, blocked line-of-sight, interference,..- attract not
Samuel Žák, Martin Kopkáš, Peter Ševčík, Martin Húdik University of Žilina, Univerzitná 8215/1, 01026 Žilina, Slovakia, Email: \{samuel.zak, martin.kopkas, peter.sevcik, martin.hudik \}@fri.uniza.sk\}

so many realizations. The possibility of energetic selfsufficiency - as of the WSN biggest asset - omission, due to the unlimited energy sources indoors, makes utilization of WSNs indoors a lot ineffective. However, there exist some indoor applications, where utilization of application-specific WSNs can be advantageous.

In [19], authors introduced a smart home system which could supervise household appliances remotely and realize real-time monitoring of home security status through mobile phone offering the possibilities of real-time monitor of the house status. In [20], the paper described a practical design and implementation of WSN for controlling and monitoring system in multi-storey building implemented in the university building. Authors in [21] proposed a service oriented architecture for development of an enterprise networking environment that was used for integrating facilities management applications and building management systems for the purpose of the enterprise environment control. The application of WSN brought in this case significant benefits due to the lack of wiring installations allowing flexible positioning of the sensors, especially when building retrofitting was concerned. In [22], the paper presented a WSN-based smart monitoring and control system for building automation. The system was developed with focus on significant reduction of energy consumption of the building under control. The data gathered by WSN were used in order to calculate and estimate the requirements for heat corrections, with respect to ventilation and weather predictions. Non-traditional usage of Facebook as a platform for such kind of indoor sensor network monitoring system was examined in [23]. For the purposes of building emergency-management system, an indoor emergence guidance algorithm based on WSN to guide people to a building exit gate while helping them avoid a hazardous area was introduced in [24]. WSN was responsible for real-time monitoring of the environment, when emergency event was detected the proposed algorithm was applied. The proposed guiding mechanism was successfully examined and verified through the real test bed implementation. 
Developments in the field of WSNs, coming out from the MEMS system designs advancements, allow realizing special WSN-based-systems for smart grids too. Smart grid technology is one of the recent developments in the area of electric power systems that aid the use of non-conventional sources of energy in parallel with the conventional sources of energy [25]. By realizing the two-way communication between the utility and the smart meters in the houses, smart grid enables a time-of-use tariff to reduce peak load through incenting residents to adopt a more efficient usage of domestic appliances [26]. However, by application of specific WSN-based smart grids, implementation of local networks, allowing bidirectional communication of local distribution grid with related electric meter. Reliable and, especially, more efficient power metering could be in this case reached. Some applications of WSN-based smart grids can be seen in [25], [26], [27] or [28], where different application scenarios were discussed: e.g. the designs of power sensing nodes to calculate the power for any kind of loads; the designs of special energy management schemes connecting non-urgent appliances to the smart meter through the wireless sensor networks; the architecture of reliable communication protocol for the harsh WSN-based smart grids environment; the architecture of WSN as a platform to enable detailed household monitoring interfaced with smartgrid through a single input to the building control system.

\section{SHARED-POWER SOURCES METERING}

In this paper, the problematic of local power metering at special places with the shared power meters and, therefore, shared bills for power sources, is targeted. Places with shared power sources, where electricity usage is calculated equally according to the number of users, are often misused by single users for illegitimate power usage. The creativity of the individuals is unlimited. The people are able to risk their health to spare a little amount of money modifying the installations to provide the possibilities of power feeding for their gadgets. The most common case is to extend the pure lighting installations with sockets, where their rarely used devices, which have to run permanently, are attached, e.g. freezers, wine coolers, servers, battery chargers, heaters(!)... These devices do not require such a high power, so the circuit breakers can cope with this load. In the Fig. 1 can be seen the typical "cheating" scenario.

The problem, which was described above, was bother the users of public garages in Slovakia, housing estate "Vlčince" in Žilina (Fig. 2), where people paid exceptional amount of money for their garages "taking advantage" of shared power metering. The particular garages were equipped just with garage door openers - power consumption under load around $400 \mathrm{~W}$, in standby only $1 \mathrm{~W}$ and with neon-tube lamps - 20W - but the average daily power consumption was after re-counting on each user $3 \mathrm{kWh}^{-1}$ year, what means $830 \mathrm{Wh}^{-1} /$ day per common user which opens and closes the garage doors in 30 s two times a day.
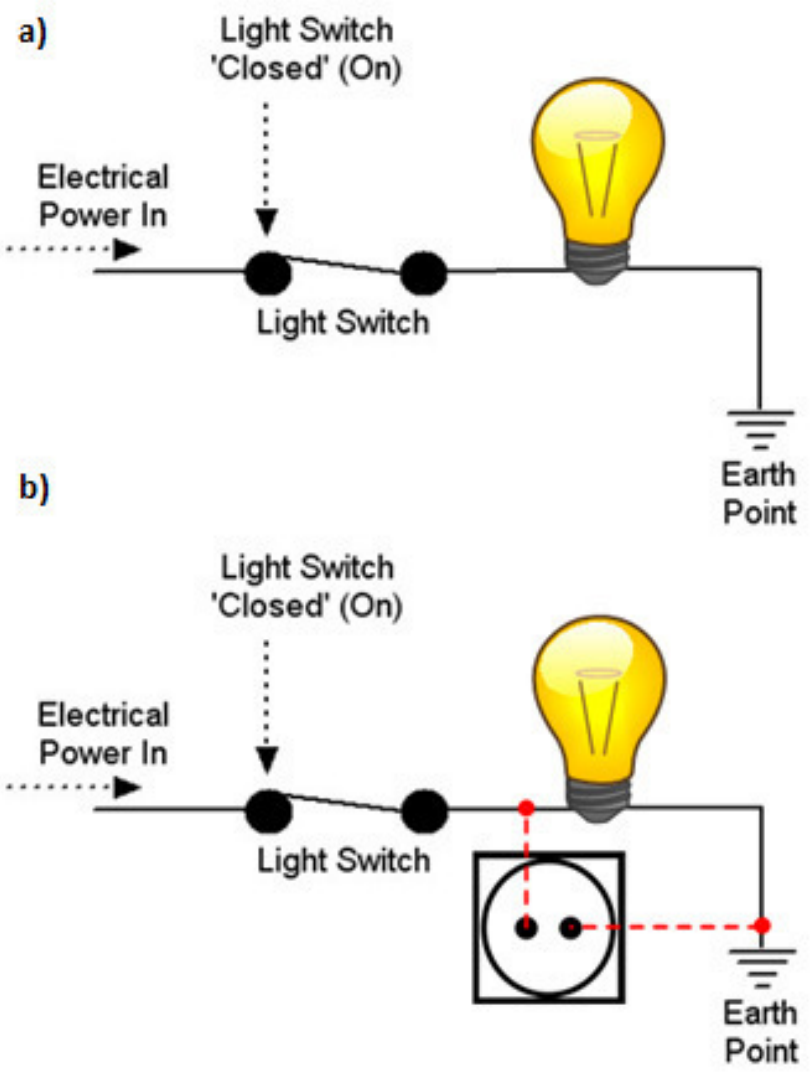

Fig. 1 a) normal lighting installation b) modification of normal lighting installation extended by power socket for "black" power consumption

Therefore the inspection of particular garages was performed to investigate the quality of electrical installations - not every user wanted to make the garage accessible.

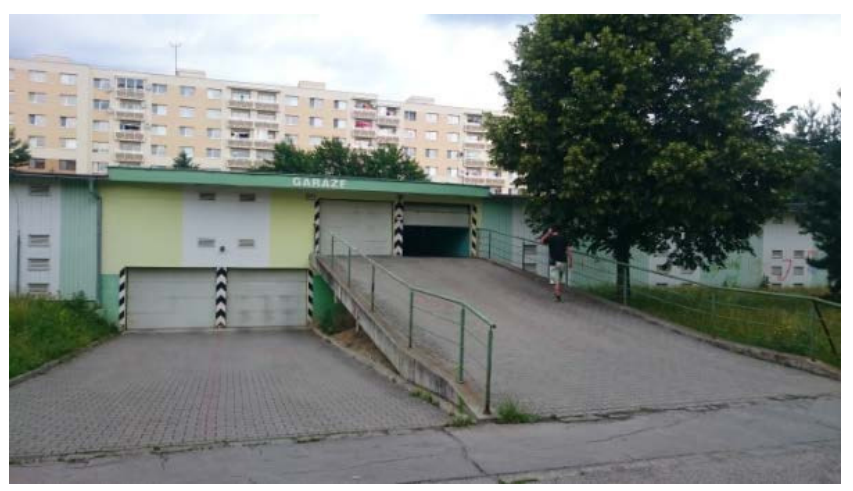

Fig. 2 Investigated garage house

The reason was trivial, "black" power sockets were presented in some garages. The garage owners modified the installations and used the power sockets for illegitimate power usage. In the figures below can be seen the installation modifications. 


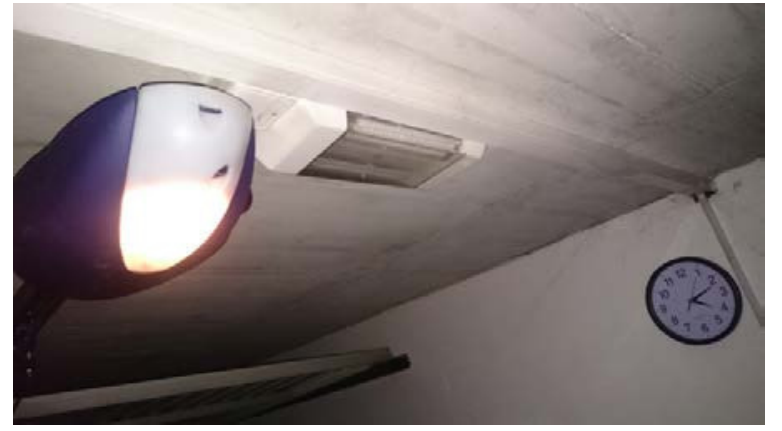

Fig. 3 Lighting installation as the source of shared power

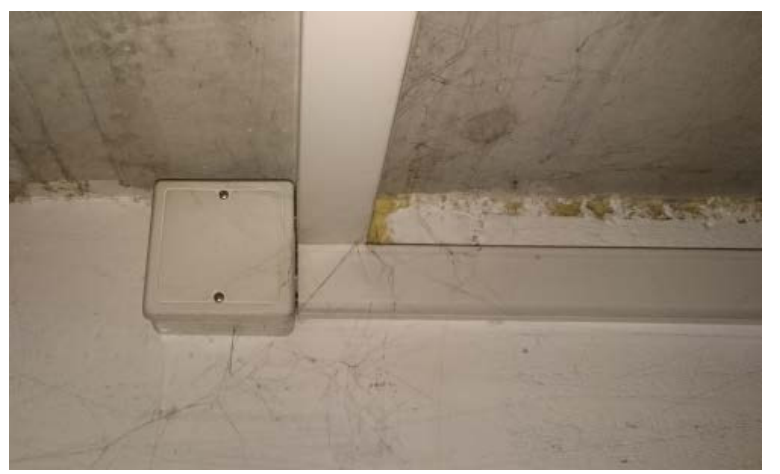

Fig. 4 Modification of lighting installation by screw terminal

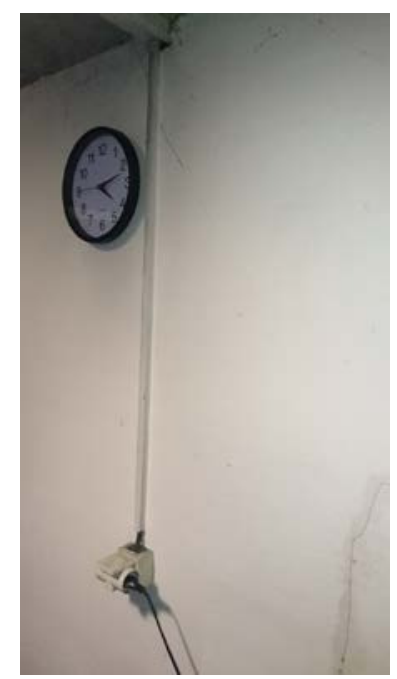

Fig. 5 Illegal socket as a "black" extension of lighting installation

To help people to overcome this problem, the special WSNbased power grid monitor was introduced. The power grid monitor is a system for energy consumption monitoring in the electricity grid. It consists of a measuring device in the form of the outlet adapter and the display device. The outlet adapter task is to measure the mains voltage and current that passes through it. The measured data are sent wirelessly to the display device. The outlet adapter consumes no more than $30 \mathrm{~mW}$ during the measurement and it is able to measure with $15 \mathrm{kHz}$ sampling rate (it means 300 samples per period $(20 \mathrm{~ms}))$. The outlet adapter is able to turn on / off the output which allow controlling remote devices, e.g. external lighting, or black power consumption characterized by increased power consumption. Moreover, switching can be automated on the time basis, power consumption or the outlet adapter temperature. So it can be used as a fuse.

As a display device with API, there is currently used a personal PC with its own wireless module in the form of a USB key. Wireless module communicates with the outlet adapter in unlicensed bands at frequencies around $868 \mathrm{MHz}$. Wireless range is $200 \mathrm{~m}$ in ideal conditions, in real about half. The outlet adapter can be upgraded to have wired data connection, but currently does not have this opinion. USB acts in this case as an interconnection gateway providing connection to the other users (admin) with particular nodes employing the star architecture.

\section{A. The outlet adapter}

The outlet adapter device is capable of measuring mains voltage and current drawn from the output of the outlet adapter. This output can be turned on / off via the communication interface. The device has a wireless communication interface for unlicensed bands around $868 \mathrm{MHz}$. Wired interface is not implemented yet, but there is this option. Also "Power-line" communication can be added, it means to transfer data directly via the electricity grid.

Possible use of the outlet adapter:

- Electricity meter - The device can measure the power consumption and report it to the remote computer. The graphical application on the computer evaluates the power consumption of multiple outlet adapters.

- Electronic fuse - The device can monitor current limit and turn off output when the limit is exceeded. Turn off limit can be set in application. The device should be turn on again after a time period so no manual turn on is needed.

- Remote device switching - User can through a computer with a wireless module freely turn on / off the device plugged into the outlet adapter. This is an advantage especially for the inaccessible devices e.g. Christmas lights in an exterior or on the roof.

- Power inverter management - Modern household equipped with a solar panel and power inverter can use consumption information so that the inverter will only supply the power that the household consummates (and charge the batteries with the rest power). So the household wouldn't become energy producer and could be resistant to short power dropout. During the power dropout the device will separate the household and the rest of the electricity grid. The inverter would then supply the household or a part of it.

Present version of the socket, which will be in our case installed at each garage doors power input is shown in a Fig. 5. To secure the socket, the socket cover is connected to the $\mathrm{I} / \mathrm{O}$ pins of the socket, so the operator knows when the cover 
was removed. Therefore, if the cheater would like to somehow manipulate with the particular socket, it could be easily identified, since each socket will be accessible only from particular garage interior. A newer version of the device could be in a DIN rail housing thus could be put between circuit breakers.

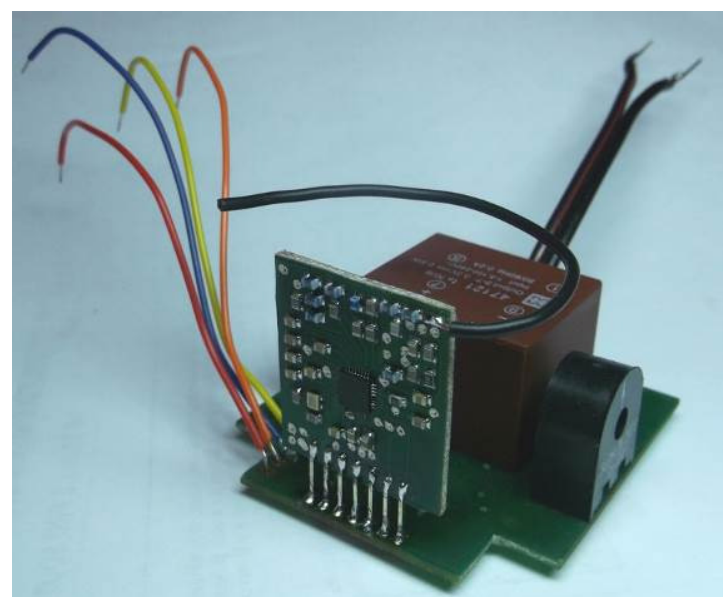

Fig. 6 The outlet adapter, system socket

\section{B. Wireless Module for PC}

Small module as a USB key contains a wireless transceiver with built-in antenna. The module transmits in the unlicensed bands, the same as the outlet adapter. Communication radius is approximately 200 meters in ideal conditions; in the building it is a bit less (depending on the particular location). The primary usage of the module is for receiving measured data from the individual outlet adapters. User application installed on a computer can interpret this data (e.g. consumption graph plotted for each outlet adapter for a certain period, displaying current / average consumption...). The USB module is shown in the Fig. 6.

The modules are able to communicate well with each other, allowing the interconnection of computers over a greater distance as Wi-Fi network. The transmitter can be improved in order to reach the communication radius near to $1 \mathrm{~km}$. The disadvantage is the low communication speed only $12 \mathrm{kbps}$. Other possible applications could be for warehouses or logistics centers where computers are far apart and need to transfer only a small amount of data.

\section{Description OF THE SYSTEM HW DESIGNS}

Outlet adapter is using MSP430AFE252 microcontroller, which has a differential 24-bit sigma-delta converter with two input channels (there are also versions with more channels). Input voltage range for the converter is $+-0,5 \mathrm{~V}$. One channel measures the electric grid voltage on the voltage divider adapted for its input range. The second channel measures the voltage at the current transformer "AX-1000" from the manufacturer "Talema". Mains conductor passes through the transformer to the device connected to the outlet adapter. The voltage at the transformer is thus proportional to the current flowing through the device (current includes also its own consumption). The maximum operating current which can microcontroller converter measure is 10A. Output from the power latching relay flows "JSL-D5N-K" manufacturer "Fujitsu". The relay is used to control the output of the two coils, which are activated by a voltage of $5 \mathrm{~V}$. The maximum power that can load the component part was set by the manufacturer to $2000 \mathrm{~W}$.

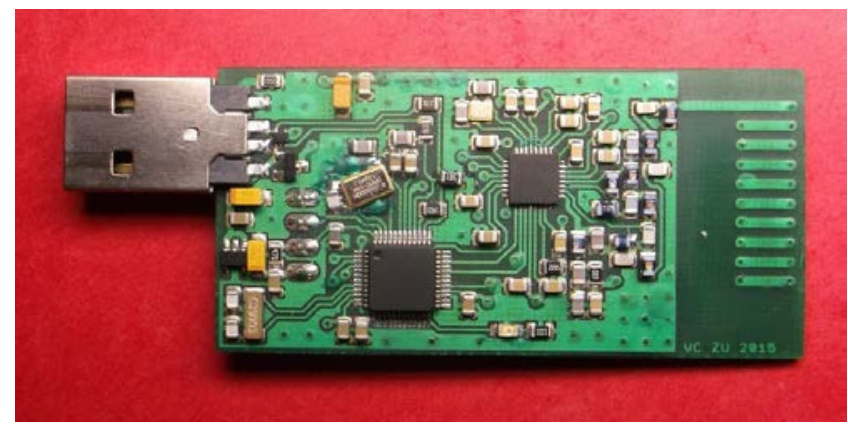

Fig. 7 USB wireless module for PC, interconnection gateway

Outlet adapter electronics is powered from the switching power supply from "Myrra" producer. The switching power supply changes the AC mains to stabilised operating voltage $3,3 \mathrm{~V}$ with a maximum current consumption $750 \mathrm{~mA}$. Producer guaranteed that average efficiency is $65 \%$ for this switching power supply. We did not observe any malfunctioning during its working. The outlet adapter includes a charge pump "TPS60401" manufacturer "Texas Instruments". The charge pump is connected as a voltage inverter and serves as a power supply for the bistable relay.

Changing the voltage using the charge pump is advantageous for this application. The main reason is its high efficiency and low circuit complexity. Low output current may seem like a disadvantage because of the higher current necessary to change the relay status. Usage of the bistable relay however means that the control current in the coils may not stay for the conservation of the relay. Its switch requires only short pulse. Since the relay is the only component that uses a negative potential, it does not matter if it is this tension fluctuate. To switch the relay is sufficient to have a negative voltage branch of a sufficiently large capacity of which short pulse will pass.

The wireless transmitting and receiving is provided through "CC1120" device from "Texas Instruments". The module combines transmitter with maximum output of $16 \mathrm{dBm}$ and receiver sensitivity of $-123 \mathrm{dBm}$ at a $1,2 \mathrm{kbps}$. Important features are summarized in the following points:

- Operating voltage is from $2 \mathrm{~V}$ to $3.6 \mathrm{~V}$. 
- 4-wire communication SPI bus and an interrupt signal.

- Special FIFO memory for transmitting and for receiving, both 128 bytes.

- Maximum transmission power to $50 \mathrm{Ohm}$ antenna varies depending on the supply voltage. For $2 \mathrm{~V}$ power supply is $12 \mathrm{dBm}$ and for $3.6 \mathrm{~V}$ is $16 \mathrm{dBm}$

- Power consumption when in max+imum power transmitting mode $(+15 \mathrm{dBm})$ is $50 \mathrm{~mA}$. Power consumption for receiving mode is approximately $22,6 \mathrm{~mA}$.

- The transmission frequency rate can be 164$192 \mathrm{MHz}, 274-320 \mathrm{MHz}, 410-480 \mathrm{MHz}$ or $820-$ $960 \mathrm{MHz}$

- Width of the transmission channel can range from $7812 \mathrm{~Hz}$ to $200 \mathrm{kHz}$.

- Circuit supports the following modulation: FSK-2, 2-GFSK, 4-FSK, 4-GFSK, ASK, OOK, DSSS and analog FM

- The maximum bit rate is half of the width of the transmission channel. For the two-state frequency modulation is the highest bit rate of $100 \mathrm{ksps}$. When four state modulation it can be equal to the width of the channel - thus maximum 200kbps.

Wireless module offers the useful function "Enhanced Wake on Radio" (eWOR). This is a power-saving mode of receiving when the module turns on and off wireless module at regular intervals to detect the presence of the transmitter. This process reduces the average current consumption during receiving. It is possible to make the communication protocol in order to use this function by simply increasing the preamble size before the data packet so the receiver had more opportunities to capture the packet.

Wireless module CC1120 supports hardware processing of the packet that is shown in the figure below.

\begin{tabular}{|c|c|c|c|c|}
\hline Preamble & $\begin{array}{c}\text { Synchr. } \\
\text { word }\end{array}$ & $\begin{array}{c}\text { Packet } \\
\text { length }\end{array}$ & Data & CRC \\
\hline $\begin{array}{c}4-240 \\
\text { bits }\end{array}$ & $\begin{array}{c}11-32 \\
\text { bits }\end{array}$ & 8 bits & $\begin{array}{c}256 \times 8 \\
\text { bits } \\
\text { (max) }\end{array}$ & $\begin{array}{c}16 \\
\text { bits }\end{array}$
\end{tabular}

Fig. 8 Hardware packaging data into packets

The preamble does not contain any valuable data, usually only alternation of ones and zeros. The task of the preamble is to give remote devices possibility to capture the presence of a transmitted signal to wake up from standby. The data transfer can be realized even without the preamble. Next important part is the synchronization word. Its purpose is to indicate the start of transmission of important data. If the receiver does not receive proper synchronization word transmission failed. Next part is the length of the packet. Length of the packet means only the size of user data that follow this part. This part may be omitted and sets the protocol for constant packet length. The last addition is the CRC code through which the correctness of the transferred data can be checked. Wireless module calculates its own $\mathrm{CRC}$ code from the received data and compares it with the received CRC code. If during transmission, there are some interference sources, which caused data defection, data are automatically declared non-conformity and will be erased.

The transmitted data encapsulates also two bytes for addressing devices and two bytes for the security key. The current implementation of the key can be understood as a password by which the system will or will not respond to the packet.

Smart metering units have their own standardized protocol that ensures compatibility among devices from different manufacturers. It is described in the standard "EN 13757", developed by "CENELEC". Wired communication protocol is commonly referred to as "Mbus" and wireless version as "WMbus". Standard "EN 13757" contains six documents. The latest ones are from 2013. [29], [30], [31], [32], [33]

The current weaknesses of the system are mainly related to the crisis cases. As an example, a power failure in the electric grid can be considered. Power failure causes loss of measured data which have not been sent to headquarters. The biggest problem is the loss of measured consumption. There are two ways to prevent it. The first is to use the backup battery, which provides data transmitting after power failure. This case assumes that the central has its own backup battery. The advantage of this method is the possibility of error detection in electrical distribution. If the mains voltage re-starting, headquarter can detect the status of the measuring devices. If the device reports the absence of voltage, it is situated in the faulty branch. The second way how to deal with data loss in case of power failure is using of non-volatile memory in which can the node hold the regularly stored data. In case of failure by using of such equipment, only a small part of data record will not be recorded.

Headquarter for the measuring device presents a personal computer. Ability to communicate in the ISM band at a frequency below $1 \mathrm{GHz}$ gives the USB device. For proper operation of the device to a computer it is necessary to add a custom driver. Subsequently, the device appears as a virtual serial link. The easiest way is through the usage of direct communication from serial line.

The main scope of the WSN implementation was to distinguish the difference between authorized and 
unauthorized power consumption. An analysis of power characteristic waveforms will help with this problem, since it provides the information about the load type.

\section{POWER FACTOR ESTIMATION}

The definition of the power factor (PF) in AC circuits is well-known as the ratio of the real power $\boldsymbol{P}$ that is used to do work and the apparent power $\boldsymbol{S}$ that is supplied to the circuit:

$$
P F=\frac{P}{\| s \rrbracket},
$$

where $\boldsymbol{P F}$ is power factor, $\boldsymbol{P}$ is real power in watts [W] and $|\boldsymbol{S}|$ is apparent power - the magnitude of the complex power in volt amps [VA].

The power factor can get values in the range from 0 to 1 . When all the power is reactive power with no real power (usually inductive load) - the power factor is 0 . When all the power is real power with no reactive power (resistive load) the power factor is 1 .

For sinusoidal current, the power factor $\boldsymbol{P F}$ is equal to the absolute value of the cosine of the apparent power phase angle $\varphi$ :

$$
P F=|\cos \varphi|,
$$

where $\varphi$ is the apparent power phase angle.

In order to estimate power factor, we can compute real energy $\boldsymbol{E}_{\boldsymbol{P P}}$ consumed during one period by formula:

$$
E_{p p}=\left(\sum_{i=0}^{n-1} U_{i} \cdot I_{i} \times T_{g}\right) \text {, }
$$

where $\boldsymbol{U}_{\boldsymbol{i}}$ in [V] and $\boldsymbol{I}_{\boldsymbol{i}}$ in [A] are sampled values during one period of mains voltage, $\boldsymbol{T}_{\boldsymbol{s}}$ is the sampling period in [s] and $\mathrm{n}$ is number of samples sampled during one period of mains voltage.

Now we can estimate apparent energy $\boldsymbol{E}_{\boldsymbol{S P}}$ as:

$$
E_{\text {sp }}=U_{\text {RuS }} \cdot I_{\text {RuS }} \cdot T_{s} \text {, }
$$

We can get $\boldsymbol{U}_{\boldsymbol{R} M \boldsymbol{S}}$ and $\boldsymbol{I}_{\boldsymbol{R} M \boldsymbol{S}}$ as the maximal sampled value divided by the square root of two:

$$
\begin{aligned}
& U_{\text {RMS }}=\frac{\mid{\mathrm{v}_{\text {MAXX }} \mid}_{\sqrt{2}},}{I_{\text {RMS }}=\frac{\left|I_{\text {MAX }}\right|}{\sqrt{2}},}
\end{aligned}
$$

where $\boldsymbol{U}_{\boldsymbol{M A X}}$ and $\boldsymbol{I}_{\boldsymbol{M A X} \boldsymbol{X}}$ are absolute maximums of sampled waveforms.

When we have all this values we can compute power factor $\boldsymbol{P F}$ by the formula:

$$
P F=\frac{E_{D D}}{E_{S D}},
$$

In normal case the light bulb has resistive character. Therefor the power factor is very close to 1 . In case of inductive (or capacitive) load the power factor is lower. This indicates that the lessee uses forbidden type of appliance. Differences between reactive and resistant power waveforms can be seen in the figures below.

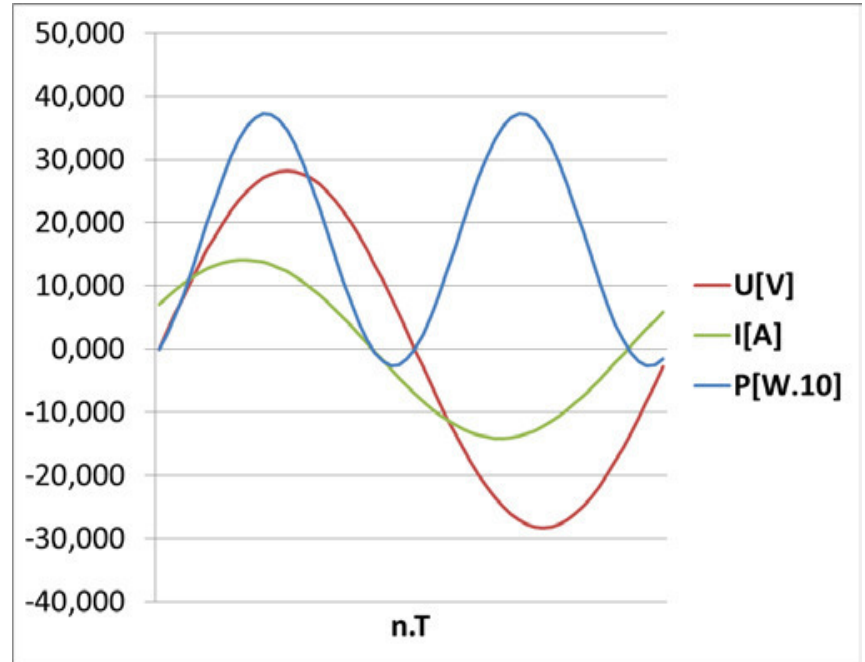

Fig. 9 Waveform of reactive power characteristics

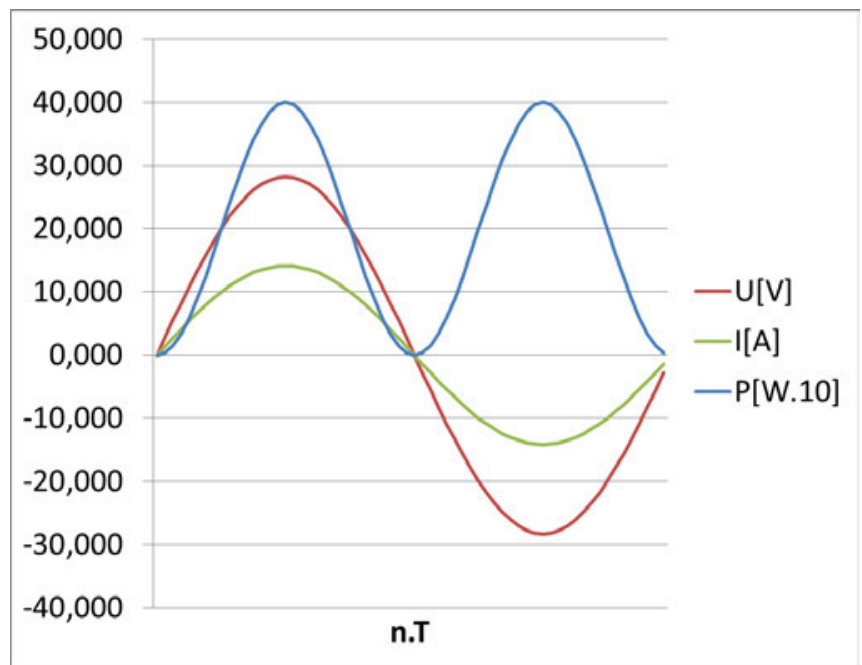

Fig. 10 Waveform of resistant power characteristics

\section{V.CONCLUSION}

The paper described the first try to implement the application-specific indoor WSN for power metering at places with shared power sources. Practical measurement shows, that the network is ready to be finally applied. However, approval of all garage owners is necessary, what is at the moment an irresolvable problem. Other concrete application scenario has therefore to be found.

\section{ACKNOWLEDGMENT}

The research is supported by the European Regional Development Fund and the Slovak state budget for the project "Research Centre of University of Žilina", ITMS 26220220183.

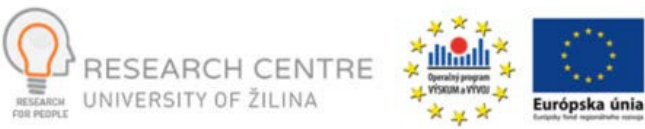

"We support research activities in Slovakia/project cofounded from the resources of the EU." 


\section{REFERENCES}

[1] M J. Miček, J. Kapitulík: WSN sensor node for protected area monitoring, FedCSIS, 2012: IEEE. - ISBN 978-83-60810-51-4.

[2] Chakravarthi, V. S.; Bhaskar, R. S.; Kusanur, V. - Conceptual Frame Work of Smart WSN for Bangalore Urban Environment Monitoring, 4th International Conference on Computational Intelligence, Communication Systems and Networks (CICSyN), 2012, Pages: 5963, DOI: 10.1109/CICSyN.2012.21

[3] Peng Yu; Xu Yong; Peng Xi-yuan - GEMS: A WSN-based greenhouse environment monitoring system - IEEE Instrumentation and Measurement Technology Conference (I2MTC), 2011, Pages: $1-6$, DOI: 10.1109/IMTC.2011.5944132.

[4] M. Hodon, P. Šarafín and P. Ševčík - Monitoring and Recognition of Bird Population in Protected Bird Territory, ISCC 2015, The Twentieth IEEE Symposium on Computers and Communications, Larnaca, Cyprus, 06.-09.July.

[5] J. Papán, M. Jurecka, and J. Púchyová - WSN for forest monitoring to prevent illegal logging, In: FedCSIS: Proceedings of the Federated conference on computer science and information systems. pp. $809-$ 812. Wroclaw, 2012, Poland.

[6] Ning Jin; Renzhi Ma; Yunfeng Lv; Xizhong Lou; Qingjian Wei - A novel design of water environment monitoring system based on WSN, International Conference on Computer Design and Applications (ICCDA), 2010, Year: 2010, Volume: 2, Pages: V2-593 - V2-597, DOI: $10.1109 /$ ICCDA.2010.5541305

[7] J. Miček, O. Karpiš, V. Olešnaníková and M. Kochlán̆ - Monitoring of Water Level Based on Acoustic Emissions, ISCC 2015, The Twentieth IEEE Symposium on Computers and Communications, Larnaca, Cyprus, 06.-09.July 2015.

[8] O. Karpiš, J. Juríček and J. Micek - Application of wireless sensor networks for road monitoring, In 10th IFAC workshop on programmable devices and embedded systems, 2015, Vol. 3. pp. 611617.

[9] M. Hodoň, M. Chovanec and M. Hyben -Intelligent traffic-safety mirror, In: Studia informatica universalis - ISSN 1621-7545. - Vol. 11 , no. 1 (2013), online, pp. 87-101.

[10] R. Žalman, J. Kapitulík and M. Kochláň - Distributed Sensor Network for Vehicles with Prior Right Detection, The Twentieth IEEE Symposium on Computers and Communications, Larnaca, Cyprus, 06.-09.July 2015

[11] R. Žalman, J. Milanová - Analysis and synthesis of acoustic signal in transport systems, CSIT 2014, Lviv, Ukraine. ISBN 978-617-607669-8. - p. 166-167.

[12] J. Furtak, J. Chudkiewicy - The concept of authentication in WSNs using TPM, Proceedings of the 2013 IEEE Federated Conference on Computer Science and Information Systems, pp. 183 - 190, 978-14673-4471-5.

[13] J. Púchyová, M. Kochláň, and M. Hodoň - Development of Special Smartphone-Based Body Area Network: Energy Requirements, Proceedings of the 2013 IEEE Federated Conference on Computer Science and Information Systems, pp. 895-900, 978-1-4673-4471-5.

[14] M. Kochlan, and P. Sevcik - Supercapacitor power unit for an eventdriven wireless sensor node, In: Federated Conference on Computer Science and Information Systems (FedCSIS), 2012 Publication Year: 2012 , Page(s): $791-796$

[15] O. Karpiš: Solar-cell based powering of a node for traffic monitoring, IOSR journal of engineering, 2013. - ISSN 2278-8719.

[16] T. Bernard, and H. Fouchal - Slot Scheduling for Wireless Sensor Networks, In: IOS Press, Journal of Computational Methods in Science and Engineering, doi:10.3233/JCM-2012-0432
[17] V. Olešnaníková and J. Púchyová - Analysis of voice activity detection for implementation into WSN. CSIT 2014, Lviv, Ukraine. ISBN 978-617-607-669-8. - p. 75-76.

[18] M. Hodoň et al. - Maximizing Performance of Low-Power WSN Node on the Basis of Event-Driven-Programming Approach, ISCC 2015, The Twentieth IEEE Symposium on Computers and Communications, Larnaca, Cyprus, 06.-09.July 2015.

[19] Y. Zhai, X.Cheng - Design of Smart Home Remote Monitoring System Based on Embedded System, 2nd International Conference on Computing, Control and Industrial Engineering (CCIE), 2011 IEEE(Volume:2 ), 20-21 Aug. 2011, Page(s): 41 - 44, ISBN: 978-14244-9599.

[20] Vo, Minh-Thanh, Tran, Van-Su ; Nguyen, Tuan-Duc ; Huynh, HuuTue - Wireless Sensor Network for Multi-Storey Building: Design And Implementation, Published in: International Conference on Computing, Management and Telecommunications (ComManTel), 2013, 21-24 Jan. 2013, Page(s): 175 - 180, ISBN: 978-1-4673-2087-0

[21] Malatras, A.; Asgari, A.; Bauge, T. - Web Enabled Wireless Sensor Networks for Facilities Management, IEEE Systems Journal, Volume:2, Issue: 4, Page(s): 500 - 512, ISSN: 1932-8184

[22] Skeledzija, N., Cesic, J. ; Koco, E. ; Bachler, V. ; Vucemilo, H. N.; Dzapo, H. - Smart Home Automation System for Energy Efficient Housing, 37th International Convention on Information and Communication Technology, Electronics and Microelectronics (MIPRO), 2014, 26-30 May 2014, Page(s): 166 - 171, Print ISBN: 978-953-233-081-6

[23] Youngjin Choi et al. - Monitoring System Employing Facebook Platform for WSN, IEEE 15th International Conference on Advanced Communication Technology (ICACT), 2013, 27-30 Jan. 2013, Page(s): 1037 - 1041, ISSN: 1738-9445, ISBN: 978-1-4673-3148-7

[24] A. Adel Ali, M. Al-Shaboti, and A. Al-Zubairi - An Indoor Emergency Guidance Algorithm Based on Wireless Sensor Networks, International Conference on Cloud Computing (ICCC), 2015, 26-29 April 2015, page(s): 1 - 5, ISBN: 978-1-4673-6617-5

[25] Yerra, R. V. P.; Bharathi, A. K. ; Rajalakshmi, P. ; Desai, U. B. - WSN Based Power Monitoring in Smart Grids, 7th International Conference on Intelligent Sensors, Sensor Networks and Information Processing, 6-9 Dec. 2011, p: 401 - 406, Print ISBN: 978-1-45770675-2

[26] Peng Han ; Jinkuan Wang ; Yinghua Han ; Qiang Zhao - Novel WSNBased Residential Energy Management Scheme in Smart Grid, IEEE International Conference on Information Science and Technology (ICIST), Page(s): 393 - 396, 23-25 March 2012, ISBN: 978-1-45770343-0

[27] Sahin, D; Bulbul, S.; Gungor, V.C; Kocak, T. - Reliable Routing in Wireless Sensor Networks for Smart Grid Environments, Published in: 20th Signal Processing and Communications Applications Conference 2012, 18.04.-20.04 2012, p(s): 1 - 4, E-ISBN: 978-14673-0054-4, Print ISBN: 978-1-4673-0055-1

[28] A. Marchiori - Enabling Distributed Building Control with Wireless Sensor Networks, IEEE International Symposium on a World of Wireless, Mobile and Multimedia Networks, Page(s): 1 - 3, 20-24 June 2011, ISBN: 978-1-4577-0352-2

[29] EN 13757-4:2013: Communication systems for meters and remote reading of meters - Part 4: Wireless meter readout (Radio meter reading for operation in SRD bands)

[30] UM1759 User manual Wireless M-Bus firmware and application, June 2014 DocID026279 Rev 1, available on-line at http://www.st.com/st-web-ui/static/active/jp/resource/technical/ document/user_manual/DM00115100.pdf

[31] http://www.ti.com/tool/WMBUS

[32] EN 13757-2:2004: Communication systems for and remote reading of meters - Part 2: Physical and link layer

[33] EN 13757-3:2004: Communication systems for and remote reading of meters - Part 3: Dedicated application layer 